\title{
Kalirin, a Multifunctional Rho Guanine Nucleotide Exchange Factor, Is Necessary for Maintenance of Hippocampal Pyramidal Neuron Dendrites and Dendritic Spines
}

\author{
Xin-Ming Ma, Jianping Huang, Yanping Wang, Betty A. Eipper, and Richard E. Mains \\ Department of Neuroscience, University of Connecticut Health Center, Farmington, Connecticut 06030-3401
}

\begin{abstract}
The structures of dendritic spines and the dendritic tree, key determinants of neuronal function, are regulated by diverse inputs that affect many scaffolding and signaling molecules. Nevertheless, here we show that reduced expression of a single gene results in loss of dendritic spines and a decrease in dendritic complexity. Kalirin, a dual Rho GDP-GTP exchange factor, causes spine formation when overexpressed. Reduced expression of Kalirin in CA1 hippocampal neurons resulted in a reduction in linear spine density, with dispersion of postsynaptic density markers and elimination of presynaptic endings. Simplification of the apical dendritic tree preceded simplification of basal dendrites. Pyramidal cell axons were not dramatically altered. Although many factors determine dendrite shape and spine formation, expression of Kalirin is necessary for the normal function of these many regulatory elements.
\end{abstract}

Key words: Kalirin; hippocampus; dendrite; spine; guanine nucleotide exchange; antisense

\section{Introduction}

Neuronal function is determined in part by factors that control the morphology of the dendritic tree, which is critical for receiving, processing, and integrating synaptic inputs. Understanding neuronal plasticity requires understanding the many factors that control the actin cytoskeleton (Schmidt and Hall, 1998; Luo, 2002). Among these factors are the Rho GTPases, small GTPbinding proteins activated by guanine nucleotide exchange factors (GEFs) of the Dbl family and inactivated by GTPaseactivating proteins (GAPs) (Luo, 2002; Quilliam et al., 2002). Racl, RhoA, and Cdc42 are highly expressed in the hippocampus, playing central roles in the specification of dendritic morphology (Olenik et al., 1997; Nakayama et al., 2000). Among the mammalian GEFs, only Kalirin and Trio have multiple Rho GEF domains, allowing them to interact in a regulated manner with multiple Rho proteins. The single Trio/Kalirin homolog in Drosophila (dTrio) plays an essential role in axonal pathfinding (Luo, 2000). When overexpressed in neurons, Kalirin and Trio stimulate outgrowth of neuronal processes (Penzes et al., 2001a,b; Estrach et al., 2002). Although several proteins, notably Homer, Shank, and SPAR (spine-associated RapGAP), are crucial to spine growth and maturation (Pak et al., 2001; Sala et al., 2001; Ehlers, 2002), to date, only Kalirin has been shown to increase the linear density of spine-like structures (Penzes et al., 2001a, 2003).

Received Aug. 1, 2003; revised Sept. 20, 2003; accepted Sept. 23, 2003.

This work was supported by National Institutes of Health Grants DK-32948 and DA-00266. We thank Darlene D'Amato for many aspects of laboratory support, Dr. Francesco Ferraro and Rich Johnson for creating vectors, Dr. Steve Potashner and Dr. Sanoj Suneja for the use of their tissue chopper, Dr. Matt Rasband for antibodies, and Lei Jin for helping us make dissociated hippocampal cultures.

Correspondence should be addressed to Richard E. Mains, Department of Neuroscience, University of Connecticut Health Center, 263 Farmington Avenue, Farmington, CT 06030-3401. E-mail: mains@uchc.edu e-mail. Copyright $\odot 2003$ Society for Neuroscience $\quad$ 0270-6474/03/2310593-11\$15.00/0
Kalirin was first identified by its interaction with peptidylglycine $\alpha$-amidating monooxygenase (PAM), an enzyme essential for neuropeptide biosynthesis (Alam et al., 1996). In addition to its two GEF domains, Kalirin has a kinase domain and several protein-protein interaction domains (Fig. $1 A$ ). Alternative splicing of the Kalirin gene generates many transcripts encoding functionally distinct proteins that are highly expressed in neurons in a tissue-specific and developmentally regulated manner (Johnson et al., 2000; Ma et al., 2001). Kal-9 and Kal-12, dual Rho GEFs, are highly expressed during embryonic development (Hansel et al., 2001). Overexpression of Kal-9 in sympathetic neurons produces axon initiation and elongation (May et al., 2002).

Kal-7 is the most prevalent Kalirin isoform in the adult rat brain (Johnson et al., 2000; Penzes et al., 2000). Kal-7 is concentrated in the postsynaptic density fraction, where it interacts with several PDZ [postsynaptic density-95 (PSD-95)/Discs large/zona occludens-1] domain-containing proteins (Penzes et al., 2000, 2001a). Overexpression of Kal-7 caused the formation of spinelike structures in cortical neurons (Penzes et al., 2001a). The increase in spine density induced by ephrin B1 was blocked by a Kal-7 mutant lacking GEF activity, by a dominant negative mutant of Rac1, or by a peptide inhibitor of Pak (Penzes et al., 2003). Although these data show that Kalirin can affect process initiation and outgrowth and spine formation, overexpression studies cannot discern what role Kalirin normally plays in these processes.

Therefore, we sought a system in which antisense methods could be used to assess the consequences of reducing levels of endogenous Kalirin. The predictable dendritic morphologies of hippocampal pyramidal neurons and the availability of excellent systems for studying hippocampal neurons led us to select this system to explore the role of endogenous Kalirin (Nakayama et al., 2000; Tashiro et al., 2000). Hippocampal pyramidal neurons 
express high levels of Kalirin, and expression increases after electroconvulsive shock, suggesting a role for Kalirin in subsequent structural changes (Ma et al., 2002). Antisense methods were used to decrease levels of endogenous Kalirin in isolated hippocampal neurons in an otherwise normal milieu, causing a marked reduction in spine density followed by progressive simplification of the dendritic tree.

\section{Materials and Methods}

\section{Animals and tissue preparation}

Timed pregnant adult female Sprague Dawley rats from Charles River Laboratories (Wilmington, MA) were housed one per cage with a 14/10 $\mathrm{hr}$ light/dark cycle with food and water available ad libitum. All experiments were conducted in accordance with the guidelines established by the University of Connecticut Health Center Animal Care and Use Committee.

For in situ hybridization, brains from postnatal day 1 (P1), P7, P14, and P30 rats were rapidly frozen on dry ice. Coronal sections $(16 \mu \mathrm{m})$ through the hippocampus were cut with a cryostat and mounted on poly-L-lysine-coated slides. The Kalirin spectrin probe encoded part of the spectrin-like region common to all forms of Kalirin [nucleotides (nt) 2684-3377, GenBank accession number AF232669]. The Kal-7 and Kal-12 probes were specific to the 3' ends of Kal-7 (nt 4903-5008, GenBank accession number AF230644) and Kal-12 (nt 8395-8905, GenBank accession number AF232669). High-specific activity antisense and sense riboprobes were made from the appropriate pBluescript-SKII $(-)$ plasmid using [ $\left.{ }^{35} \mathrm{~S}\right] \mathrm{UTP}$ with $\mathrm{T} 7$ or T3 polymerase, respectively. In situ hybridization was performed as previously described (Ma et al., 1999); notably, all sections from different ages in a time course were hybridized at the same time and were exposed to film or nuclear emulsion for equal times.

For immunohistochemistry and immunocytochemistry, rats were anesthetized with ketamine and perfused transcardially with saline followed by $4 \%$ paraformaldehyde in PBS. Coronal sections $(12 \mu \mathrm{m})$ were cut and mounted on gelatin-coated slides. The specificity of the Kal spectrin antibody was previously determined (JH2582, 1:1000; Ma et al., 2001). Kal-7 and Kal-12 polyclonal antisera were affinity-purified (Penzes et al., 2001a); specificity was evaluated by blocking with preincubation of antibody with antigen $(10 \mu \mathrm{g} / \mu \mathrm{l})$. In addition, Western blot analysis revealed proteins of the expected masses. After overnight incubation at $4^{\circ} \mathrm{C}$ with primary antibody diluted in a block, sections were processed for staining with a rabbit $\mathrm{ABC}$ kit (Vector Laboratories, Burlingame, CA).

Hippocampal slices were fixed for $1.5 \mathrm{hr}$ in $2.5 \%$ formaldehyde containing $4 \%$ sucrose in $1 \times$ PBS and then immersed in $30 \%$ sucrose $(\mathrm{w} / \mathrm{v}$ in $1 \times$ PBS) for $2 \mathrm{hr}$. After a quick freeze on dry ice, slices were thawed, rinsed in $1 \times$ PBS for $5 \mathrm{~min}$, and then processed as described for tissue. Slices were stained simultaneously with affinity-purified polyclonal antisera to Kal-7 (1:100) or Kal-12 (1:100) and a monoclonal antibody to green fluorescent protein (GFP, 1:100; Chemicon, Temecula, CA); primary antibodies were visualized with Cy3-labeled donkey anti-rabbit IgG (Jackson ImmunoResearch, West Grove, PA) and FITC-labeled goat anti-mouse IgG (Jackson ImmunoResearch).

\section{Western blot analysis}

Western blot analyses were performed as described (Johnson et al., 2000). Hippocampi were homogenized in radioimmunoprecipitation assay buffer (Johnson et al., 2000), and protein concentrations were determined using the BCA reagent with bovine serum albumin as the standard (Pierce, Rockford, IL). Proteins were visualized using an Amersham Biosciences (Arlington Heights, IL) ECL Kit with affinity-purified primary antisera to Kal-7 or Kal-12 (both at 1:200).

\section{Primary cultures of rat hippocampal neurons}

Hippocampi from embryonic day 18 Sprague Dawley rats were separated from diencephalic structures, chopped, and digested with $0.28 \%$ trypsin (Invitrogen, San Diego, CA) for $25 \mathrm{~min}$ at $37^{\circ} \mathrm{C}$ with gentle shaking. Dissociated cells were plated at low density $\left(1 \times 10^{4}\right.$ cells $/ \mathrm{ml}$ in $35 \mathrm{~mm}$ glass-bottom microwell dishes coated with poly-L-lysine or in $35 \mathrm{~mm}$ dishes with poly-L-lysine-coated $12 \mathrm{~mm}$ coverslips) or at high density $\left(2 \times 10^{5}\right.$ cells $/ \mathrm{ml}$ onto coated coverslips). Cells were plated in Neurobasal A medium containing 7\% heat-inactivated horse serum, $1 \times$ B27 supplement, $0.5 \mathrm{~mm}$ glutamine, and $25 \mu \mathrm{M}$ 2-mercaptoethanol and maintained at $37^{\circ} \mathrm{C}$ in a $5 \% \mathrm{CO}_{2}$ atmosphere (all reagents from Invitrogen). Three hours later, plating medium was replaced with fresh medium containing only $3 \%$ horse serum. One day after plating, the culture medium was exchanged with Neurobasal A medium containing 2\% B27 supplement, $0.5 \mathrm{~mm}$ glutamine, $25 \mu \mathrm{M} 2$-mercaptoethanol, $50 \mathrm{U} / \mathrm{ml}$ penicillin, and $50 \mu \mathrm{g} / \mathrm{ml}$ streptomycin (Invitrogen). Thereafter, $50 \%$ of the medium was replaced twice a week for up to 4 weeks.

\section{Organotypic rat hippocampal slice cultures}

Slice cultures were made as described (Gainer et al., 2002). Hippocampi from P8 Sprague Dawley rats were dissected into ice-cold sterile Gey's balanced salt solution (Sigma, St. Louis, MO) containing 0.5\% glucose. Medial hippocampi were sliced transversely $(400 \mu \mathrm{m})$ using a Mcllwain tissue chopper. Slices were kept in ice-cold Gey's balanced salt solution and then placed onto $30 \mathrm{~mm}$ Millicell CM membrane inserts (Millipore, Bedford, MA) in Petri dishes containing $1.1 \mathrm{ml}$ of culture media composed of $0.5 \times$ Eagle's basal medium, $0.25 \times$ HBSS, $0.25 \times$ horse serum (defined, heat-inactivated), $25 \mathrm{U} / \mathrm{ml}$ penicillin, $25 \mu \mathrm{g} / \mathrm{ml}$ streptomycin, and $1 \mathrm{~mm}$ L-glutamine (Invitrogen). Slices were kept under a $5 \% \mathrm{CO}_{2}$ atmosphere at $37^{\circ} \mathrm{C}$, with medium changes at $1 \mathrm{~d}$ in vitro (DIV) and every $3 \mathrm{~d}$ thereafter.

\section{Expression vectors and biolistic transfection}

The pCMS dual-promoter vector (Clontech, BD Biosciences, Palo Alto, CA) was engineered so that GFP was expressed under control of the SV40 promoter. Expression of the cDNA of interest was under control of the cytomegalovirus (CMV) promoter.

pCMS-GFP-Kal-GEF1. The Kal-GEF1 vector used previously (May et al., 2002) was cut with NcoI, blunted with Klenow, cut with NotI, and inserted into pCMS cut with EcoRI, blunted, and cut with NotI. This yields a $\mathrm{His}_{6}$-myc-Kal-GEF1 construct under the control of the CMV promoter (May et al., 2002).

pCMS-GFP-Kal-antisense. PCR primers were designed to excise the fragment encoding $E^{738}$-LFHERK to KEYGE- ${ }^{1150}$ of Kalirin-7 with NotI-SpeI and XhoI-KpnI linkers, respectively, inserted first into pBluescript for sequencing and then cut with XhoI and NotI for insertion into the same sites in pCMS. This region was chosen to extend from the C-terminal third of spectrin repeat 5 to the middle of spectrin repeat 9 (Alam et al., 1997), encompassing a region that is $\sim 70 \%$ identical to Trio at the amino acid and nucleotide levels, with no stretch larger than $14 \mathrm{nt}$ of exact match (McPherson et al., 2002). These sequences were selected for this study to be the most likely to block all Kalirin expression for full-length and $\Delta$-Kalirins yet to have the least potential for crossreaction with the corresponding Trio sequences. When prohormone convertases 1 and 2 were studied with a similar antisense approach (Bloomquist et al., 1991; Paquet et al., 1996), this level of difference between family members ensured complete specificity.

pCMS-GFP-Trio-antisense. The sequence $\mathrm{E}^{707}$-AQSQMEE to WIHDNG-E ${ }^{1100}$ of rat Trio was derived by PCR (as for the Kal-7381150 sequence) using pPC86.rat Trio1.3, a PAM interactor identified from a rat pituitary cDNA expression library during a yeast two-hybrid screen (R. C. Johnson, M. L. Lukas, X. M. Ma, B. A. Eipper, and R. E. Mains, unpublished data), and inserted into pBlueScript for sequencing. The pCMS-Trio-antisense (AS) vector was constructed as for the Kal-AS vector.

pCMS-GFP-PAM-antisense. The sequence from $\mathrm{I}^{176}$-SAFRDN to DHDRKL-E ${ }^{906}$ was excised from rat PAM- 1 cDNA with EcoRV and XhoI and inserted into pCMS in the antisense direction.

Plasmid DNA was purified on CsCl gradients or using Maxi-Prep kits (Qiagen, Hilden, Germany). Plasmid DNA was precipitated onto $1.0 \mu \mathrm{m}$ gold microcarrier particles at a concentration of $1 \mu \mathrm{g}$ of plasmid DNA/mg of gold, according to the Bio-Rad (Hercules, CA) instruction manual. Plasmid-coated gold particles were resuspended in $0.05 \mathrm{mg} / \mathrm{ml}$ polyvinylpyrollidone in ethanol and dried onto Gold-Coat tubing $(25 \mathrm{mg}$ gold particles/29 inches of tubing). Dissociated hippocampal cultures 


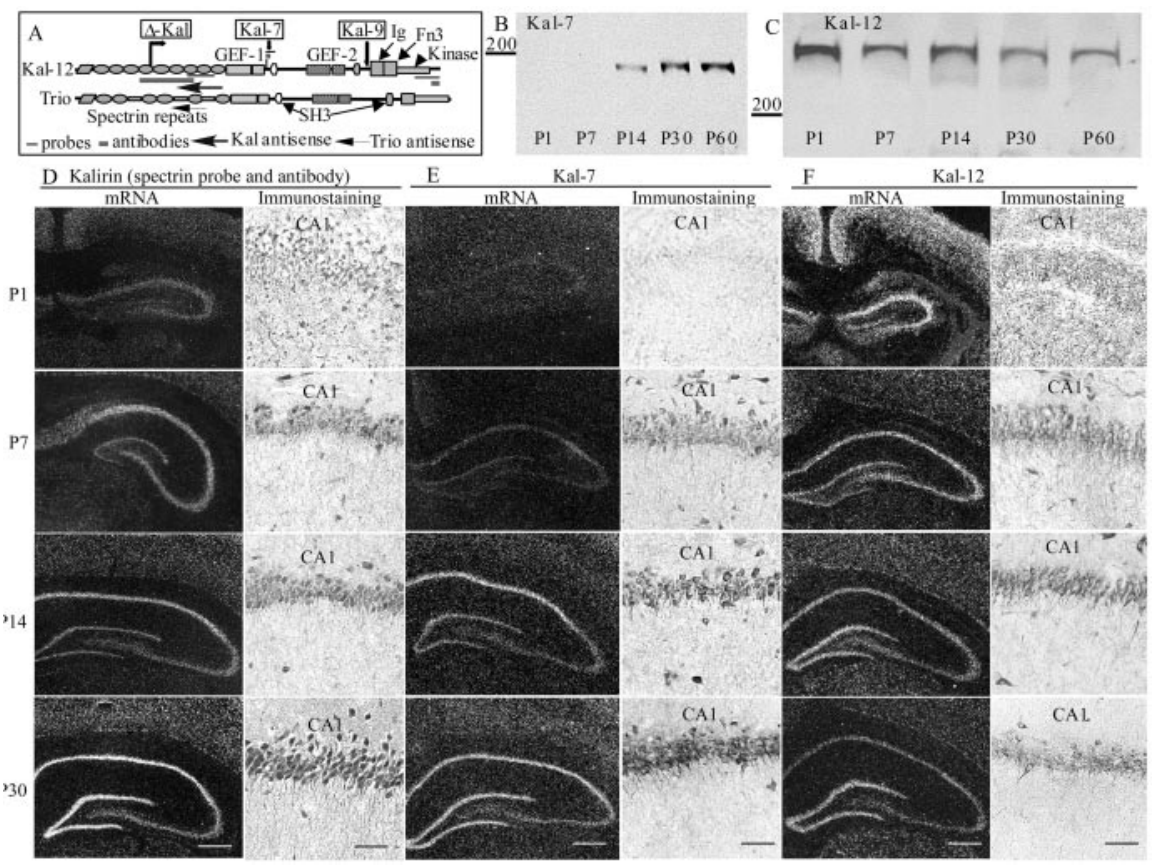

Figure 1. Developmental changes in Kalirin expression. $A$, Kalirin probes and antibodies. Kal-12 has multiple spectrin-like repeats, two Rho GEF domains, two SH3 motifs, Ig and fibronectin III motifs, and a kinase domain; unique regions in the major Kalirin is oforms are drawn to scale. Bars indicate the regions to which antibodies or probes were made. $B$, C, Hippocampal extracts (50 $\mu \mathrm{g}$ of protein) prepared from rats of the indicated ages were subjected to Western blot analysis using the affinity-purified Kal-7 and Kal-12 antibodies; molecular weight markers are shown (in kilodaltons). Tissue from rats of the indicated ages was analyzed by in situ hybridization and immunostaining with probes and antibodies specific for the spectrin-like region of Kalirin (D), Kal-7 (E), or Kal-12 (F). Kalirin mRNA and protein are expressed in all of the pyramidal neurons of CA1-CA4, dentate granule cells, and interneurons of the developing hippocampus. Scale bars: in situ hybridization, $200 \mu \mathrm{m}$; immunostaining, $100 \mu \mathrm{m}$.

and slices were transfected using a Helios Gene Gun (Bio-Rad) held 1.5-2 $\mathrm{cm}$ above the target and set at 150 psi helium pressure. Cultured neurons were generally transfected after 2-3 weeks, and hippocampal slices were transfected after 2 DIV. Biolistic transfection of slices after 0 or 1 DIV produced more pyramidal neurons with fragmented or bead-like processes that often lacked dendritic spines. Transfection after $>2$ DIV yielded more transfected glial cells, interfering with our ability to quantify the dendritic arbor of neighboring transfected neurons. For highpower images, an antibody to GFP was used to intensify the GFP signal, providing better evaluation of fine dendritic processes.

\section{Image analysis and quantification}

Isolated GFP-positive neurons were imaged using a Zeiss (Thornwood, NY) LSM510 confocal microscope. For quantification of total dendritic length, a stack of images ( $Z$ step, $1 \mu \mathrm{m}$ ) was taken using a $20 \times$ objective ( 0.8 digital zoom factor), and the entire neuron was visualized in three dimensions with Zmaris 3.2 (Bitolane AG, Zurich, Switzerland). Dendrites were traced using Neurolucida, and total dendritic length was calculated using Neurolucida. For spine quantification, stacks of confocal images (Z step, $0.5 \mu \mathrm{m}, 50-100 \mu \mathrm{m}$ from cell soma) were taken using a $63 \times$ objective ( 1.5 digital zoom factor). Three-dimensional images were assembled with Zmaris, and spines were traced and quantified using Neurolucida. Headed and headless spines were counted when no more than $3 \mu \mathrm{m}$ long (Nakayama et al., 2000). For quantification of GFP: PSD-95 clusters and GFP:Bassoon clusters, confocal images through the central focal plane were taken using a $100 \times$ objective $(1.2$ digital zoom factor). For organotypic slices, GFP-positive pyramidal neurons in CA1 were imaged using the Zeiss LSM510 microscope. For quantification of dendritic branch segments and analysis of Sholl profiles, $Z$ stacks ( $Z$ step, $1 \mu \mathrm{m}$ ) were taken using a $20 \times$ objective ( 0.8 digital zoom factor); images of the entire neuron were merged with Zmaris. For quantification of spine density, images ( $Z$ step, $0.5 \mu \mathrm{m}$ ) were taken in the area distal to the first apical branch point (usually $25-100 \mu \mathrm{m}$ from soma) or distal to the first basal branch point ( $25-75 \mu \mathrm{m}$ from the soma) using a $63 \times$ objective
(1.5 digital zoom factor). Images merged with Zmaris were traced and quantified using Neurolucida. Data are presented as average and SEM. Statistical analyses were performed with SPSS 10 (SPSS Inc., Chicago, IL). $P$ values in the text are from Student's $t$ test.

\section{Results \\ Developmental changes in Kalirin isoforms in rat hippocampus}

Before evaluating the consequences of eliminating Kalirin expression in hippocampal pyramidal neurons, we first wanted to determine which isoforms were expressed in these cells during early postnatal development. Western blot analysis of hippocampal extracts with polyclonal antisera specific for Kal-7 and Kal-12 (Fig. $1 A-C)$ revealed isoform-specific changes in expression and demonstrated the specificity of the antisera. Kal-7 is first detectable 2 weeks after birth, with levels increasing from P14 to P60; levels of Kal-12 decrease gradually from P1 to P30 (Fig. $1 B, C)$. In situ hybridization and immunostaining with reagents specific for the spectrin-like region common to most isoforms of Kalirin revealed high levels of $\mathrm{Ka}$ lirin in pyramidal neurons throughout CA1-CA4, dentate granule cells, and interneurons (Fig. 1D). In the CA1 region, Kalirin immunostaining is detected in the soma and fine dendrites of all pyramidal neurons (Fig. 1D, P30).

Using probes specific for Kal-7 and Kal-12, distinctly different expression patterns are observed. Consistent with Western blot analysis, Kal-7 mRNA levels increase dramatically during postnatal development, reaching peak levels after P14 (Fig. 1E). In contrast, Kal-12 mRNA levels decrease gradually (Fig. $1 F$ ). Expression of both isoforms is high in pyramidal neurons, dentate granule cells, and scattered interneurons. Immunostaining with antibodies specific for Kal-7 and Kal-12 confirmed these developmental changes, with Kal-7 staining increasing and Kal-12 staining decreasing during postnatal development (Fig. 1E,F). At $\mathrm{P} 14$ and P30, Kal-7 is expressed at similar levels in all CA1 pyramidal neurons.

\section{Elimination of Kalirin expression in isolated neurons}

As observed previously in dissociated cortical neurons, overexpression of Kal-7 increases the linear density of dendritic spines in dissociated hippocampal neurons (data not shown) (Penzes et al., 2001a). To determine whether Kalirin was necessary for spine formation, we needed to be able to reliably eliminate Kalirin expression in identified neurons in an otherwise normal environment. To ensure coexpression of the GFP marker and Kalirin antisense RNA, a dual-promoter vector was used (Fig. 2A). The SV40 promoter was used to drive expression of GFP, and the stronger CMV promoter was used to drive expression of the Kalirin antisense construct. The antisense RNA targets spectrin-like repeats 6-9 (amino acids 738-1150), a region common to all of the major Kalirin isoforms but readily distinguishable from Trio (Fig. 1A). We used biolistic transfection so that we could eliminate Kalirin expression in isolated neurons, focusing on cellautonomous effects. In slice cultures, hippocampal pyramidal 
neurons maintain their in vivo dendritic morphology, allowing ready identification of their apical and basolateral dendrites (Nakayama et al., 2000; Tashiro et al., 2000).

Hippocampal slices prepared from P8 rats were biolistically transfected with a vector encoding GFP alone (Fig. 2B) (Wellmann et al., 1999; Nakayama et al., 2000). For morphological evaluations, transfection parameters were adjusted to give an average of one to five GFP-positive pyramidal neurons in the CA1-CA3 regions of each slice (Fig. 2C); our goal was to have a small number of transfected neurons surrounded by a majority of cells expressing their normal complement of genes. To determine whether GFP and the gene of interest were reliably expressed in the same cell at the same time, hippocampal slices were biolistically transfected with a vector encoding both GFP and myctagged Kalirin-GEF1 (Fig. 2D), which is well expressed and encoded by a transcript similar in size to the Kalirin antisense RNA. Forty-eight hours after transfection, 98\% of the GFP-positive cells (green) expressed myc-tagged Kalirin-GEF1 (red; $\mathrm{n}=132$; Fig. $2 E$ ). As for cortical neurons, overexpression of Kalirin-GEF1 caused retraction of the dendritic tree, making it difficult to distinguish neurons from glia in low-power images (Penzes et al., 2001b) (Fig. 2 D). Nevertheless, it is clear that both genes encoded by the dual-promoter vector are reliably expressed in the same cell.

We next evaluated the effect of expressing Kalirin antisense RNA on levels of Kalirin protein. Because all CA1 pyramidal neurons normally express Kalirin (Fig. 1), we simply visualized Kalirin protein in slices fixed $2 \mathrm{~d}$ after biolistic transfection with the dual-promoter vector encoding GFP and Kalirin antisense RNA (Fig. 2C,F). Expression of GFP was first apparent 12-24 hr after transfection and remained detectable for $5 \mathrm{~d}$. A decrease in Kalirin protein was apparent $2 \mathrm{~d}$ after transfection with Kalirin antisense RNA (Fig. 2 F). Levels of Kalirin expression in the soma of CA1 hippocampal pyramidal neurons expressing GFP alone or GFP and Kal-AS were quantified in comparison with surrounding nontransfected neurons $2 \mathrm{~d}$ after transfection (Fig. 2G). On the basis of immunostaining intensity, levels of Kal-7 were decreased to $17 \%$ of control in neurons expressing the antisense construct and were unaffected by expression of GFP alone. Antisense-mediated effects were lost at times longer than $4 \mathrm{~d}$ after transfection, with a resumption of dendritic growth and an increase in spine formation. Thus, transfection of neurons with the Kal-AS vector reduces levels of Kalirin protein without irreversibly damaging the cells.

\section{Kalirin is necessary for maintenance of dendritic spines in dissociated hippocampal neurons}

To examine the effect of reducing Kalirin expression on spine structure, we first used high-density cultures of dissociated hip- pocampal neurons because it was simpler to observe the morphology of neurons in these cultures at high magnification than in slice cultures. Spines appear only in mature hippocampal neurons, which are not well transfected by traditional methods (Wellmann et al., 1999), so we used particle-mediated gene transfer to transfect individual neurons. After $48 \mathrm{hr}$, the cultures were fixed and visualized simultaneously with antisera to Kal-7 and to GFP (Fig. 3). In control neurons, GFP was distributed throughout the entire dendritic tree, including dendritic spines (Fig. $3 A, C)$. Staining of endogenous Kal-7 was noted in essentially all neurons and was not altered in the cells expressing GFP alone (Fig. $3 A, C$ ). In contrast, neurons expressing GFP and the Kalirin antisense RNA exhibited a dramatic decrease in Kal-7 staining, with staining that remained generally concentrated to one side of the nucleus (Fig. 3B,D). Kal-7 staining in neurons expressing GFP alone or GFP and the Kalirin antisense RNA was compared with Kal-7 staining in surrounding nontransfected neurons, revealing an antisense-mediated decrease in Kal-7 staining to $18 \%$ of levels in neighboring control neurons (Fig. 4A).

The axons and dendrites of hippocampal neurons are readily 

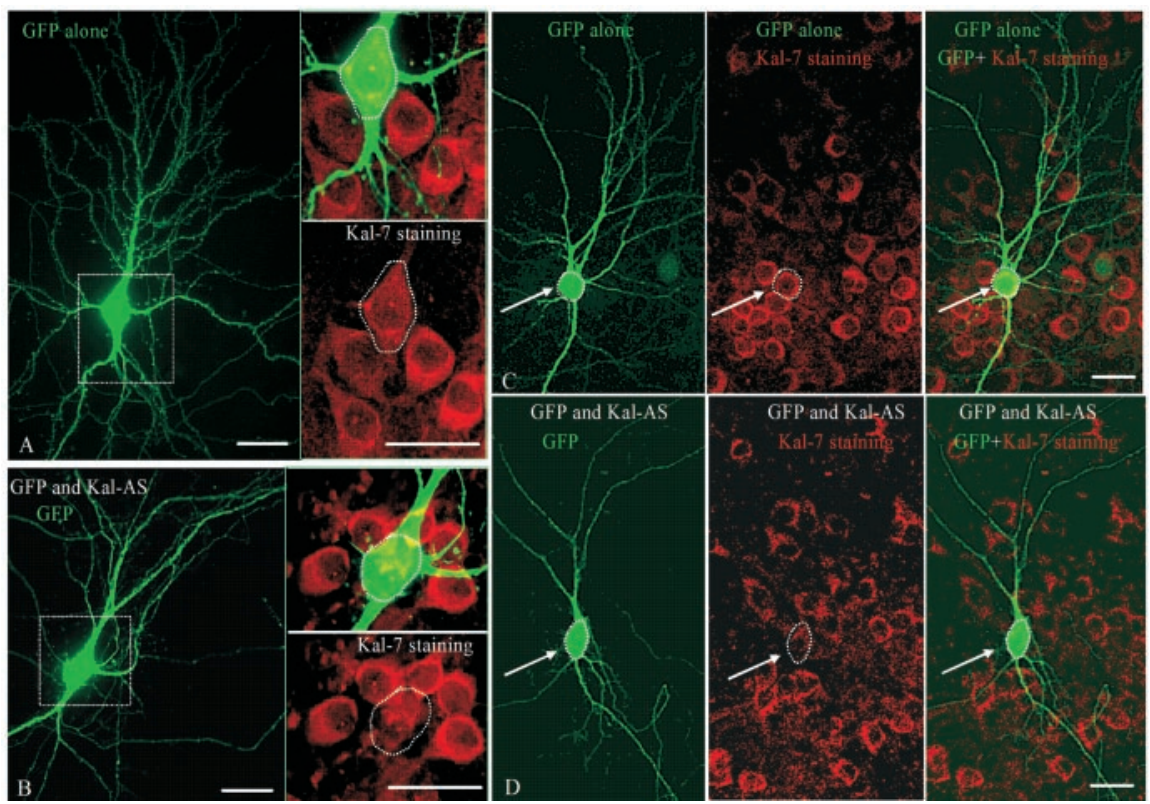

Figure 3. Reduced expression of endogenous Kalirin causes simplification of the dendritic tree and a reduction in spine density. High-density dissociated cultures of hippocampal neurons were biolistically transfected with the pCMS vector encoding GFP $(A, C)$ or GFP and Kalirin antisense RNA $(B, D)$. Cultures were fixed $48 \mathrm{hr}$ after transfection and stained simultaneously for Kal-7 (Cy3; red) and GFP (FITC; green). GFP-positive neurons in which endogenous Kal-7 expression was reduced below control levels had simplified dendritic trees. $A, B$, Insets, Higher-power images with both colors and just Kalirin staining. Scale bar, $25 \mu \mathrm{m}$.
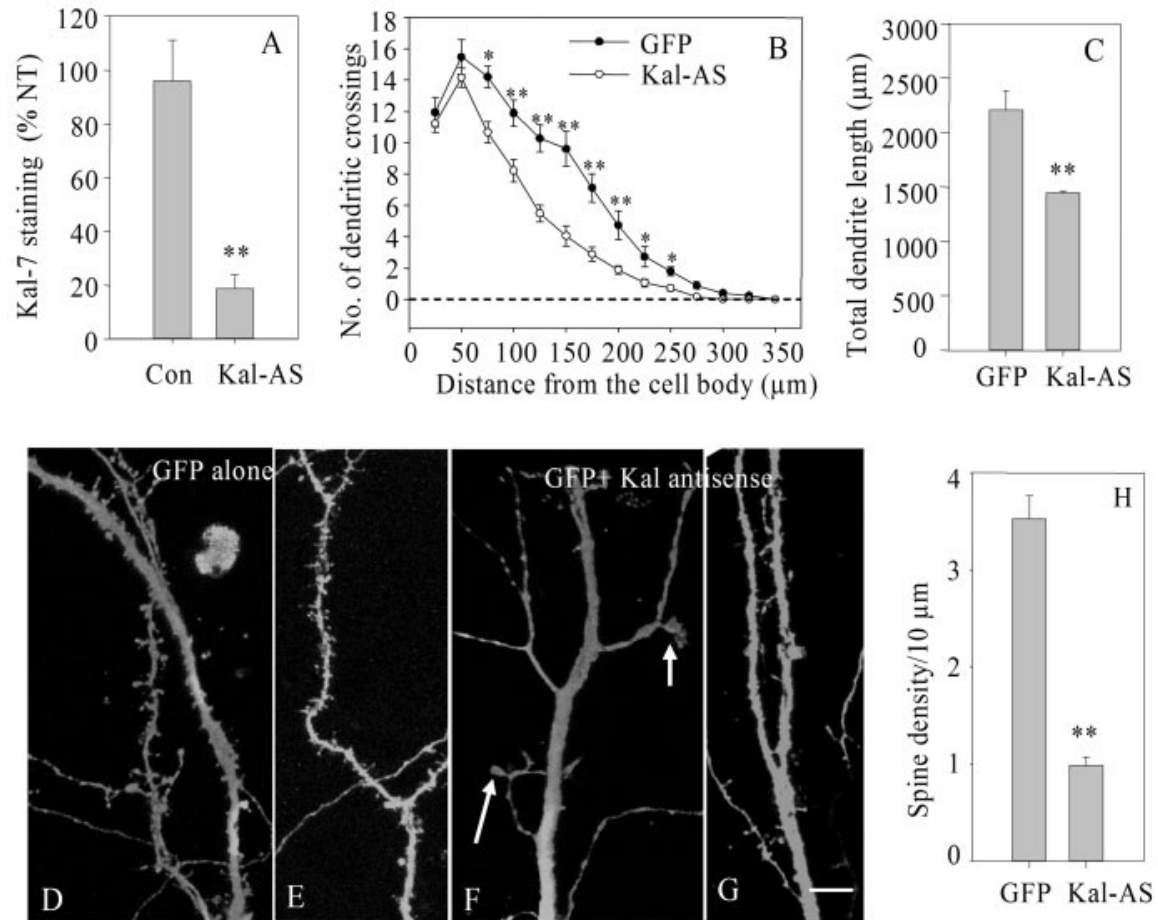

Figure 4. Quantification of the effect of decreased Kalirin expression in dissociated neurons. $A$, Using cultures prepared as shown in Figure 3, Kal-7 staining in GFP-positive control neurons (pCMS-GFP) and GFP-positive Kal-AS neurons was quantified as in Figure $2 G$ and compared with Kal-7 staining in surrounding nontransfected (NT) neurons $[n=20$ (GFP) and 36 (NT) for control; $n=19$ (Kal-AS) and 38 (NT) for Kal-AS]. Expression of Kal-7 was not affected in GFP-positive control neurons but was significantly reduced in Kal-AS neurons ( $p<0.001$, t test). $B$, Sholl analysis of neurons like those shown in Figure 3 revealed a significant difference in distal dendrites $\left(75-225 \mu \mathrm{m} ; t\right.$ test, $\left.{ }^{*} p<0.05 ;{ }^{* *} p<0.01\right)$. C, Total dendritic length was significantly reduced in Kal-AS neurons ( $t$ test, $\left.{ }^{* *} p<0.01\right)$. D-G, Higher-magnification images were acquired to examine dendritic spines; an abnormal spine-like structure is marked with arrows in $F$. Scale bar, $10 \mu \mathrm{m}$. $H$, Linear spine density was significantly reduced in Kalirin antisense neurons ( $t$ test, ${ }^{* *} p<0.01$ ). At least 20 neurons were quantified for each measured parameter. distinguished, even in a dissociated cell culture (Sampo et al., 2003). Axons are very fine and uniform in diameter and extend for long distances, whereas dendrites are tapered, branched, and shorter. Sholl analyses, which quantify the number of times the dendrites from a given neuron cross concentric circles of increasing diameter, were used to compare GFPexpressing control neurons and GFPexpressing Kalirin antisense neurons. The Sholl analyses demonstrate that reducing the level of endogenous Kalirin results in simplification of the dendritic tree (Fig. $4 B)$. Total dendrite length was decreased to $65 \%$ of control when levels of endogenous Kalirin were reduced (Fig. 4C).

Higher-magnification images were acquired to allow evaluation of spine density and morphology (Fig. 4D-G). Although the dendrites of control neurons were studded with spine-like structures (Fig. $4 D, E)$, the dendrites of Kalirin antisense neurons were often smooth, lacking any spine-like protrusions (Fig. $4 F, G$ ). When protrusions were evident, they were often longer than normal and very thin. In addition, fan-shaped protrusions were often observed (Fig. $4 F$, arrows). For quantification of spine density, a region $50-100 \mu \mathrm{m}$ from the cell soma on a major dendrite was examined. Linear spine density decreased to $28 \%$ of control in the Kalirinantisense neurons (Fig. 4H). Despite the loss of spines, few if any fibers showed the beaded appearance typical of outright degeneration.

Kalirin is necessary for maintenance of synaptic organization

We used antisera to PSD-95 (Fig. 5) and Bassoon (Fig. 6) to assess the effect of reduced Kalirin expression on synaptic organization. On the basis of costaining for MAP2, a dendritic marker, both Kal-7 and Kal-12 are localized to dendrites; neither Kal-7 nor Kal-12 colocalized with tau, an axonal marker (data not shown). Neurons expressing GFP alone (Fig. $5 A, B$ ) or GFP and the Kalirin antisense vector (Fig. $5 E, F$ ) were visualized simultaneously with antisera to PSD-95 (red), a postsynaptic marker (Cho et al., 1992), and GFP (green). As expected, almost every spinelike structure observed on the dendrites of control neurons was visualized by the PSD-95 antibody (Fig. 5A,B). In addition, as reported in several studies on PSD-95 localization (Rao et al., 1998; Sala et al., 2003; Shiraishi et al., 2003), a significant amount of PSD-95 was observed in punctate structures throughout the dendritic shaft. 
The C-terminal PDZ-binding motif of Kal-7 allows this isoform of Kalirin to interact with PSD-95 and is essential for the ability of exogenous Kal-7 to induce the formation of spine-like structures (Penzes et al., 2001a). Because our Kalirin antisense vector targets all isoforms of Kalirin, we wondered whether Kal-7 was the only isoform localized to spines. To address this issue, dissociated hippocampal neurons were stained simultaneously with polyclonal antisera to Kal-7 or Kal-12 and monoclonal antiserum to PSD-95 (Fig. $5 C, D)$. As expected, Kal-7 partially colocalized with PSD-95 (Penzes et al., 2001a) (Fig. 5C). Kal-12 exhibited a similar distribution, partially colocalizing with PSD-95 (Fig. 5D). Staining of tissue sections (Fig. $1 F)$ confirmed the presence of Kal-12 in the dendrites of hippocampal neurons at P7 and P14. As for PSD-95, significant amounts of Kal-7 and Kal-12 are localized to punctate structures in the dendritic shaft.

Neurons expressing GFP and the Kalirin antisense RNA were also visualized simultaneously with antisera to PSD-95 and GFP (Fig. 5E,F). Instead of normal spinelike structures, thinner, longer structures branched from the dendrites of the Kalirin antisense neurons. These thin structures rarely contained detectable amounts of PSD-95. Forty-eight hours after transfection, we observed a significant decrease (to $40 \%$ of control) in the linear density of PSD-95:GFP clusters in the Kalirin antisense neurons (Fig. 5G).

Staining of mature hippocampal neurons with polyclonal antisera to Kalirin and monoclonal antiserum to Bassoon, a presynaptic marker (tom Dieck et al., 1998), revealed close apposition but little colocalization of either Kal-7 or Kal-12 with Bassoon (Fig. 6A,B). Both Kal-7 and Kal-12 abutted the presynaptic terminals visualized by the Bassoon antibody. Presynaptic endings on the spines and the dendritic shaft were all visualized with antiserum to Bassoon (Fig. 6A,B). Almost every spine-like structure on the dendrites of control neurons was apposed to a Bassoonpositive structure (Fig. 6C). In contrast, the structures branching from the dendrites of Kalirin antisense neurons were not always apposed to Bassoon-positive structures (Fig. 6D). The linear density of Bassoon-positive clusters along the dendrites of Kalirin antisense neurons was significantly reduced compared with control neurons when evaluated $48 \mathrm{hr}$ after transfection (Fig. 6E). This suggests that reduced levels of endogenous
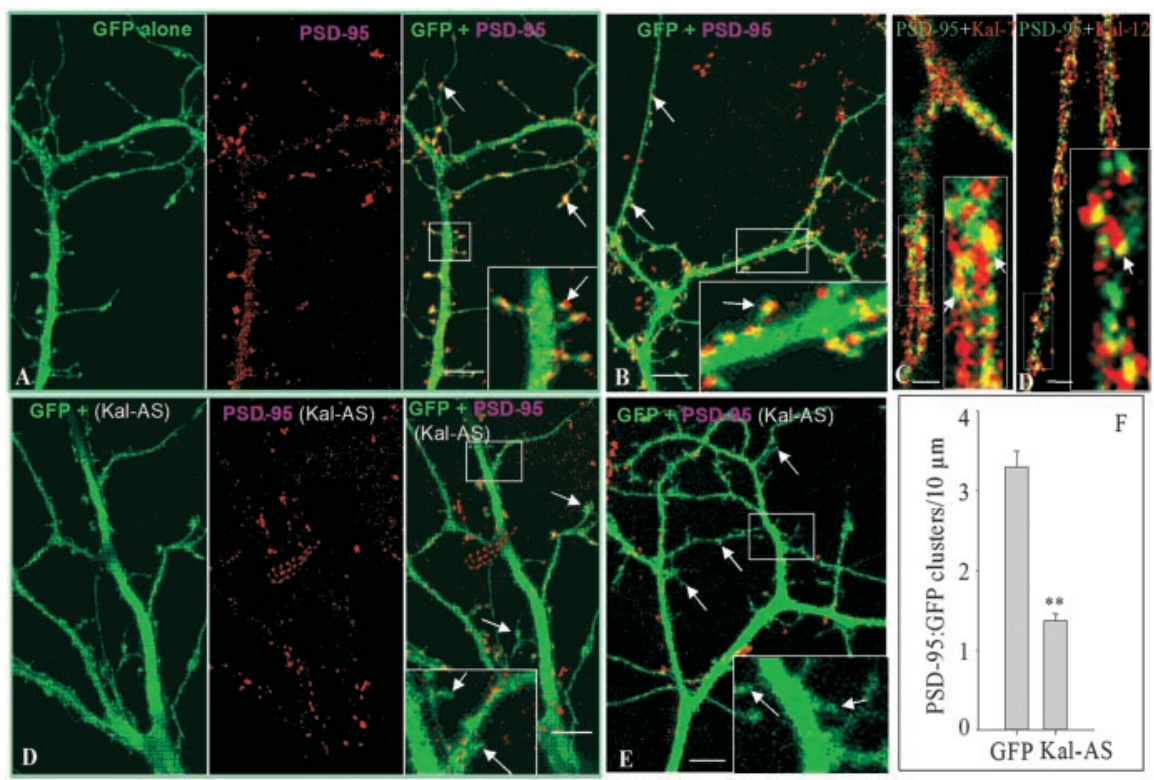

Figure 5. Postsynaptic structures are disrupted after reductions in Kalirin expression. Dissociated hippocampal neurons were biolistically transfected with the pCMS vector encoding GFP $(A, B)$ or GFP plus Kalirin antisense RNA $(D, E)$. Fixed cultures were simultaneously stained for PSD-95 (Cy3; red) and GFP (FITC; green). A, B, Insets, Arrows show overlaps of PSD-95 staining with spine tips; $D$, E, arrows show protrusions without PSD-95 staining. Scale bar, $10 \mu \mathrm{m}$. Dissociated hippocampal neurons that had not been transfected were fixed and stained simultaneously with affinity-purified antisera to Kal-7 ( $C$ ) or Kal-12 (D) (Cy3) and PSD-95 (FITC); the specificity of the affinity-purified antibodies was verified by preincubation with the appropriate peptide (image not shown). C, D, Arrows show regions of overlap for PSD-95 and Kal-7 or Kal-12, respectively. F, The number of PSD-95:GFP clusters was significantly reduced in the dendrites of hippocampal neurons expressing Kalirin antisense RNA ( $t$ test, ${ }^{* *} p<0.001$ ).
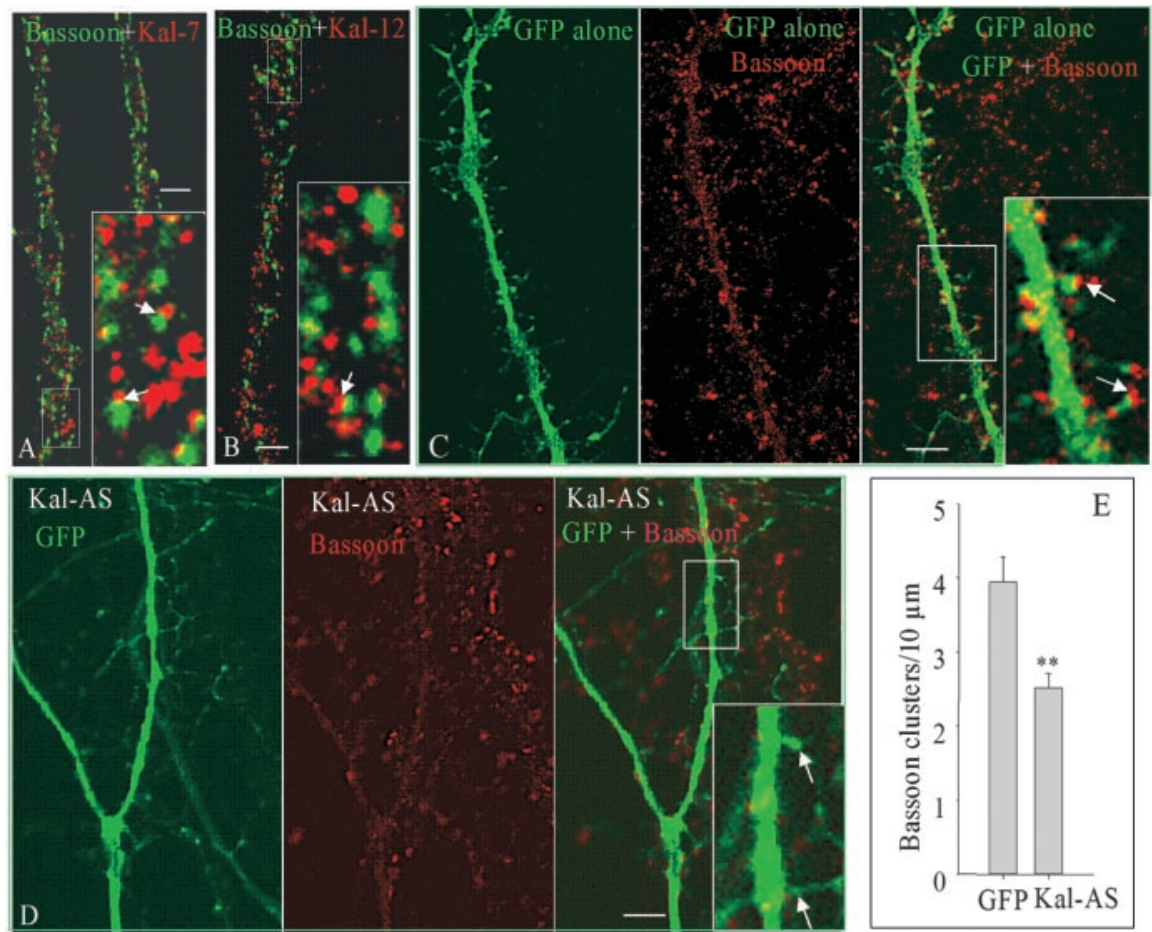

Figure 6. Presynaptic structures are disrupted after reductions in Kalirin expression. Dissociated hippocampal neurons were fixed and stained simultaneously with affinity-purified antisera to Kal-7 ( $A$ ) or Kal-12 (B) (Cy3; red) and Bassoon (FITC; green), a presynaptic marker (tom Dieck et al., 1998). Bassoon staining is apposed to the tips of dendritic spines and in puncta located on dendritic shafts. Insets, High-power magnifications of marked areas; arrows point to Bassoon (green) and Kal (red) staining. After biolistic transfection with the pCMS vector encoding GFP $(C)$ or GFP and Kalirin antisense RNA $(D)$, cultures were simultaneously stained for Bassoon (Cy3) and GFP (FITC). C, Inset, Arrows show Bassoon staining (red) at the tip of spines (green); $D$, inset, arrows point to spines (green) without bassoon staining. $E$, Neurons expressing Kalirin antisense RNA exhibited a significant decrease in Bassoon staining at clusters on dendrites ( $t$ test, $\left.{ }^{* *} p<0.01\right)$. Scale bar, $10 \mu \mathrm{m}$. 


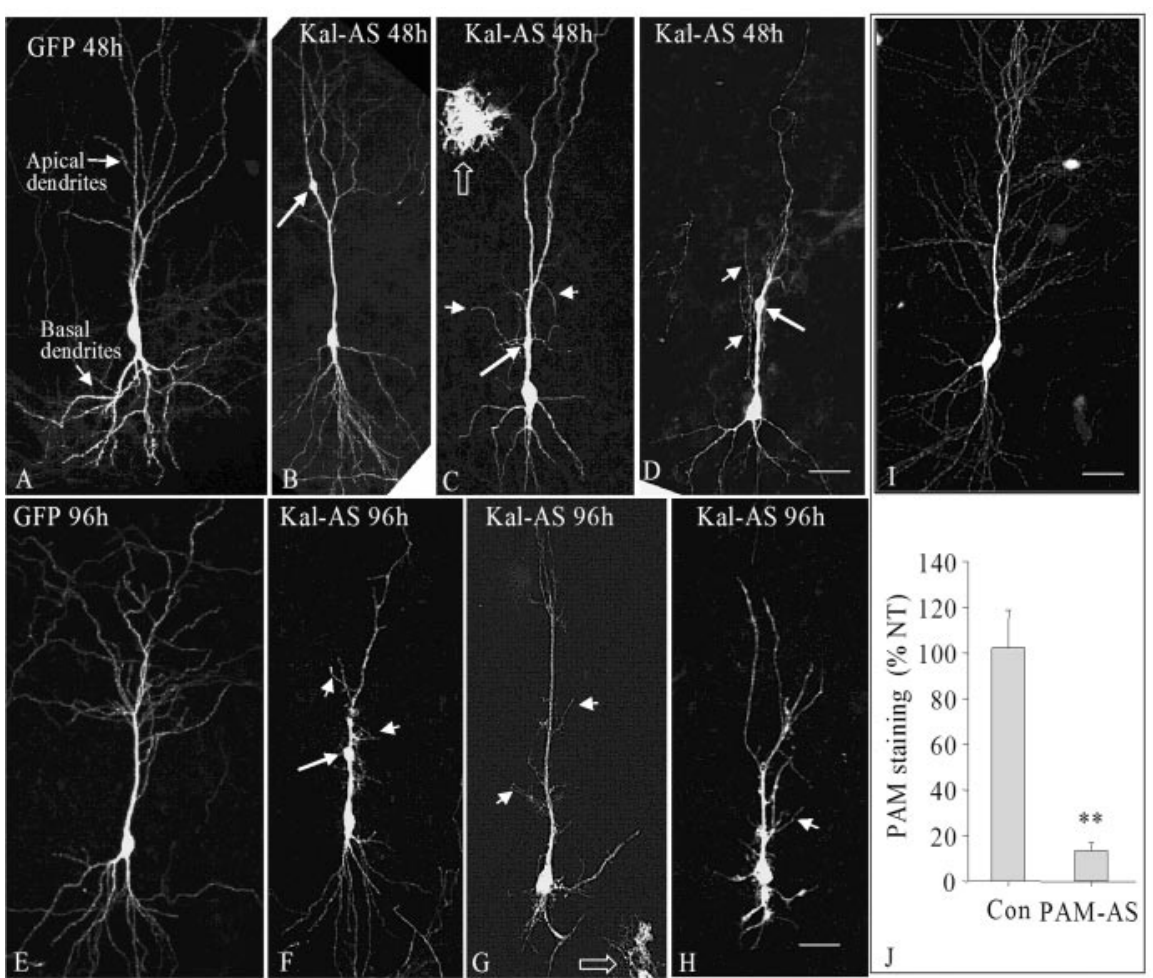

Figure 7. Expression of Kalirin-antisense RNA in hippocampal CA1 neurons results in progressive simplification of the dendritic tree. Hippocampal slices (P8) were biolistically transfected with the pCMS vector encoding GFP $(A, E)$ or GFP plus Kalirin antisense RNA $(B-D, F-H)$. Slices were fixed $48 \mathrm{hr}(A-D)$ or $96 \mathrm{hr}(E-H)$ after transfection and stained for GFP; a Z stack of confocal images was taken with a $20 \times$ objective, and the projection is shown. Bumpy protrusions (long arrows) and long, thin processes (short arrows) are marked. Scale bar, $30 \mu \mathrm{m}$. Hippocampal slices biolistically transfected with the pCMS vector encoding GFP and PAM antisense RNA were fixed after $48 \mathrm{hr}(I)$. To quantify the decrease in PAM levels, slices transfected with the $p C M S$ vector encoding GFP or GFP and PAM antisense RNA were fixed and stained for PAM; the ratio of PAM staining in GFP-positive neurons ( $n=16$ for control; $n=14$ for PAM antisense) relative to surrounding nontransfected neurons ( $n=44$ for control; $n=28$ for PAM antisense) is shown $\left(\right.$; $t$ test, $\left.{ }^{* *} p<0.001\right)$.

Kalirin in the postsynaptic neuron result in changes in the associated presynaptic terminal, presumably through a transsynaptic signaling mechanism.

On the basis of in situ hybridization and Western blot analysis, Trio is also expressed in hippocampal pyramidal neurons from P1 through P14 (data not shown). Trio is similar to the largest isoform of Kalirin, Kal-12, in gene structure (McPherson et al., 2002) (Fig. 1A). To determine whether the changes in dendritic morphology and spine density attributed to reductions in Kalirin were specific, dissociated hippocampal neurons were biolistically transfected with a pCMS vector encoding GFP and a rat Trio antisense RNA. The morphology of control and Trio antisense neurons was examined $48 \mathrm{hr}$ after transfection. Sholl analysis and spine density quantification demonstrated that there was no change in dendritic morphology. In contrast, axonal growth appeared to be disturbed, with a loss in direction and increased branching (data not shown). Because the Trio antibodies available were not sensitive enough for use in immunostaining, we could not demonstrate that expression of the Trio antisense RNA actually reduced levels of endogenous Trio. Nevertheless, these experiments demonstrate that expression of antisense RNA per se is not toxic and support the conclusion that changes in dendritic morphology brought about by the expression of Kalirin antisense RNA are specific.
Kalirin is necessary for maintenance of dendritic morphology in CA1 pyramidal neurons in hippocampal slice cultures Hippocampal pyramidal neurons maintain many of their in vivo characteristics while in slice culture (Nakayama et al., 2000). We wanted to determine whether reductions in Kalirin expression in isolated hippocampal neurons in the more complex environment of slice cultures would cause changes similar to those seen in dissociated neurons. Slices were prepared from P8 rats because hippocampal pyramidal neurons have well differentiated apical and basal dendrites by this time (Nakayama et al., 2000). To identify optimal conditions, slices were transfected with the control vector encoding GFP alone after different amounts of time in culture. The number of glial cells transfected increased when slices were transfected after longer times in culture, so slices prepared from $\mathrm{P} 8$ rats were routinely transfected after 2 DIV and fixed 48 or 96 hr later. At the time of fixation, expression of endogenous Kal-7 is just beginning to increase, and expression of Kal-12 has begun to decrease, but both isoforms are expressed in pyramidal neurons throughout CA1-CA4 as well as granule cells of the dentate gyrus and scattered interneurons (Fig. 1). Sholl profiles for the dendrites of CA1 and CA3 pyramidal neurons differ significantly, so we focused on pyramidal neurons in CA1 (Nakayama et al., 2000). The apical and basal dendrites of GFPexpressing CA1 pyramidal neurons showed little change between 48 and $96 \mathrm{hr}$ after transfection, demonstrating that the transfected neurons remained healthy (Figs. $7 A, E, 8 A, B$ ).

As shown above (Fig. $2 G$ ), expression of Kalirin antisense RNA resulted in a marked reduction in Kal-7 immunostaining in CA1 pyramidal neurons compared with neighboring GFPnegative pyramidal neurons when examined $48 \mathrm{hr}$ after transfection. The overall architecture of CA1 pyramidal neurons expressing Kalirin antisense RNA is greatly simplified (Fig. 7B-D) compared with neurons expressing GFP alone (Fig. 7A). Sholl analysis of the apical dendrites demonstrated a significant reduction in dendritic complexity after $48 \mathrm{hr}$ (Fig. $8 \mathrm{~A}$ ). Changes in the basal dendrites did not reach statistical significance at $48 \mathrm{hr}$ after transfection (Fig. 8B). When examined $96 \mathrm{hr}$ after transfection, simplification of both apical and basal dendrites was apparent (Fig. 7E vs $F-H$ ). Sholl analysis after $96 \mathrm{hr}$ showed a significant decrease in dendritic complexity for both apical and basal dendrites (Fig. $8 A, B$ ). The apical dendrites remaining on pyramidal neurons expressing Kalirin antisense RNA often had long, wispy filopodia or bumpy protrusions not observed in control neurons (Fig. $7 B-D, F-H$ ).

We were concerned that expression of antisense RNA might have a nonspecific, toxic effect. To address this concern, we created an antisense vector targeted to PAM, a gene expressed uniformly in all hippocampal pyramidal neurons at levels higher than Kalirin (Ma et al., 2002). The PAM antisense vector lowered 

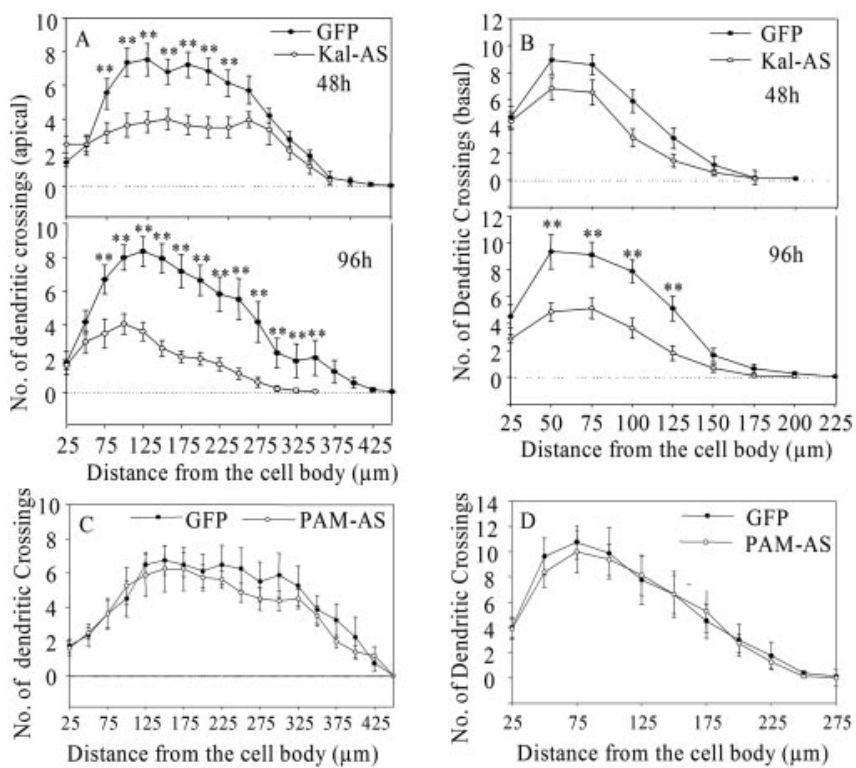

Figure 8. Quantification of the effects of expressing Kalirin or PAM antisense RNA. Sholl profiles for apical $(A)$ and basal $(B)$ dendrites in hippocampal neurons expressing GFP alone or GFP and Kalirin antisense RNA were prepared after 48 and $96 \mathrm{hr}\left(t\right.$ test, ${ }^{* *} p<0.01$ ). Similar plots were prepared for apical ( $C$ and basal $(D)$ dendrites of CA1 pyramidal neurons expressing GFP alone or GFP and PAM antisense RNA for $48 \mathrm{hr}$; no significant changes were observed.

PAM protein and enzyme activity to $50 \%$ in stably transfected cell lines overexpressing PAM, as expected from our previous studies (Mains et al., 1991). Biolistic transfection of hippocampal slices with the pCMS vector encoding PAM antisense RNA produced no change in dendritic morphology (Figs. 7I, 8C,D, Sholl plots). On the basis of immunostaining, expression of PAM antisense RNA reduced PAM staining to $<15 \%$ of control (Fig. $7 J$ ). Thus we conclude that the changes in dendritic morphology that result from expression of Kalirin antisense RNA in CA1 pyramidal neurons reflect loss of the normal function of endogenous Kalirin. As in dissociated hippocampal neurons, biolistic transfection of hippocampal slices with the pCMS vector encoding Trio antisense RNA failed to produce a change in dendritic complexity or spine density in CA1 pyramidal neurons (data not shown).

Higher-power confocal images were acquired 48 and $96 \mathrm{hr}$ after transfection to evaluate the effect of reducing Kalirin expression on dendritic spine morphology (Fig. 9). For each CA1 pyramidal neuron, images of apical dendrites were obtained distal to the first apical branch point ( $\sim 25-100 \mu \mathrm{m}$ from the cell soma), and images of basal dendrites were taken distal to the first basal dendrite branch point (25-75 $\mu \mathrm{m}$ from the soma). After $48 \mathrm{hr}$, the apical dendrites of GFP-expressing control neurons were marked with spine-like structures of fairly uniform morphology (Fig. 9A). At the same time, the dendrites of Kalirin antisense neurons were often smooth, with occasional large bulbous structures (Fig. 9B). Control GFP-expressing pyramidal neurons looked very similar after 48 and 96 hr (Fig. 9C). In many cases, Kalirin antisense neurons had only thin, filamentous structures branching from their dendrites (Fig. 9D). Few normal spine-like structures were observed. Quantitative analysis revealed a dramatic decrease in linear spine density on both the apical and basal dendrites of Kalirin antisense neurons (Fig. 9E). The effect was significantly greater $96 \mathrm{hr}$ after transfection than $48 \mathrm{hr}$ after transfection. Progressive effects of expressing Kalirin antisense RNA on the number of apical and basal dendritic segments were also observed (Fig. 9F). In contrast, expression of PAM antisense
RNA had no effect on the number of apical or basal dendritic segments (Fig. 9G).

\section{Discussion}

Kal-7 and Kal-12 have distinct roles in dendrites

Although Kal-7 and Kal-12 are expressed in the same hippocampal neurons, their distinctly different developmental time courses suggest different functions. In mature cultures of dissociated hippocampal neurons, both Kal-7 and Kal-12 are localized to the cell soma and to dendrites, not to axons. Both Kal-7 and Kal-12 are localized to the tips of spine-like structures on the dendrites of mature hippocampal neurons. In addition, both Kal-7 and Kal-12 are associated with punctate structures located within the dendritic shaft. The close apposition of Bassoon staining to the Kal-7 and Kal-12 staining at the tips of spines suggests that both are closely associated with excitatory glutamatergic synapses. Despite the similarities in immunocytochemical localization, biochemical fractionation clearly demonstrates differences; Kal-7 is enriched in the postsynaptic density fraction, but Kal-12 is not (Penzes et al., 2000). This difference is consistent with the fact that Kal-12 lacks the PDZ-binding motif that allows Kal-7 to interact with PSD-95 (Figs. 1, 10). Although their developmental time courses differ, both Kal-7 and Kal-12 are expressed in hippocampal pyramidal neurons of the age examined in our studies. Our antisense strategy eliminates expression of all of the major Kalirin isoforms in a small subset of neurons surrounded by unaffected neurons, and we did not try to distinguish among isoforms.

\section{Kalirin is necessary for maintenance of dendritic spines}

It is clear that many factors, including synaptic activity, regulate spine formation (Maletic-Savatic et al., 1999; Hering et al., 2001). At the time Kalirin expression was reduced in slice cultures, CA1 pyramidal neurons had already formed spines on their apical and basal dendrites; under control conditions, spine density was unchanged during our $4 \mathrm{~d}$ period of study. Reduction of endogenous Kalirin levels caused the progressive loss of spines from apical and later from basal dendrites of CA1 pyramidal neurons. A similar loss of dendritic spines was observed in dissociated hippocampal neurons, whereas a comparable reduction of PAM using the same antisense RNA approach did not significantly alter dendritic patterns, a crucial control for toxicity of the antisense method. Thus continued expression of Kalirin is necessary for spine maintenance. Although overexpression of Kal-7 was previously shown to cause an increase in spine density in dissociated cortical neurons (Penzes et al., 2001a), the loss of existing spines that occurred in response to decreased levels of Kalirin was not anticipated. First, both Kalirin and Trio, a closely related paralog, are expressed in the same neurons, raising the possibility of functional redundancy. Our experiments demonstrate that endogenous Trio cannot substitute for Kalirin. Trio null mice have defects in hippocampal organization, suggesting that both proteins have critical but distinct functions (O'Brien et al., 2000). Second, the existence of many neuronal Rho GEFs of overlapping specificity suggests that other GEFs might substitute for Kalirin.

A decrease in Kalirin expression triggers a sequence of events, with one of the first detectable changes being loss of PSD-95 staining on the tips of dendritic spine-like structures. Spine-like structures lacking clustered PSD-95 staining are apparent only after expression of Kalirin is reduced. At the same time, closely apposed Bassoon staining is lost, suggesting that the signaling pathways coordinating presynaptic and postsynaptic function are sensitive to loss of Kalirin. Many of the subsequent changes 

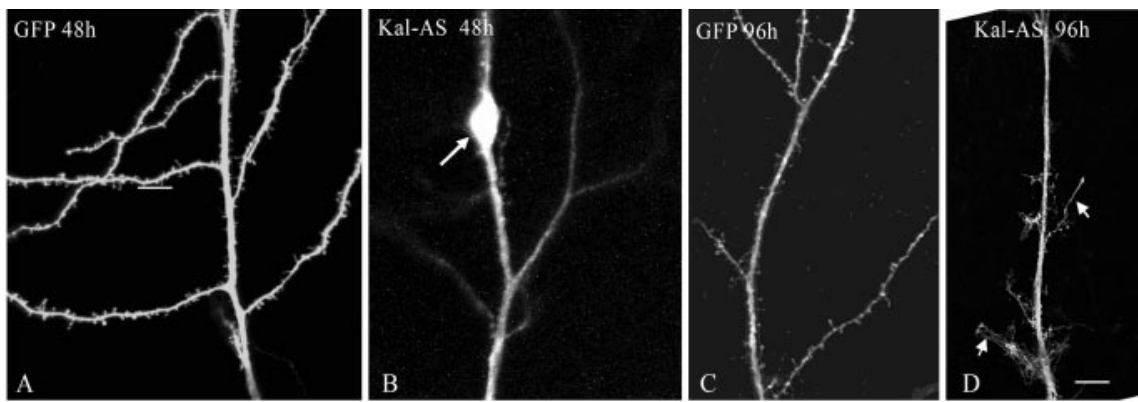

2001; Sala et al., 2001; Ehlers, 2002). Overexpression of GFP-PSD-95 stimulates increases in spine number and size (ElHusseini et al., 2000). However, targeted disruption of PSD-95 does not alter synaptic structure, possibly because of redundancy among MAGUK (membraneassociated guanylate kinase) family members. Overexpression of Shank, a multivalent scaffolding protein, acting through Homer, a multimeric adaptor, results in the formation of enlarged spines
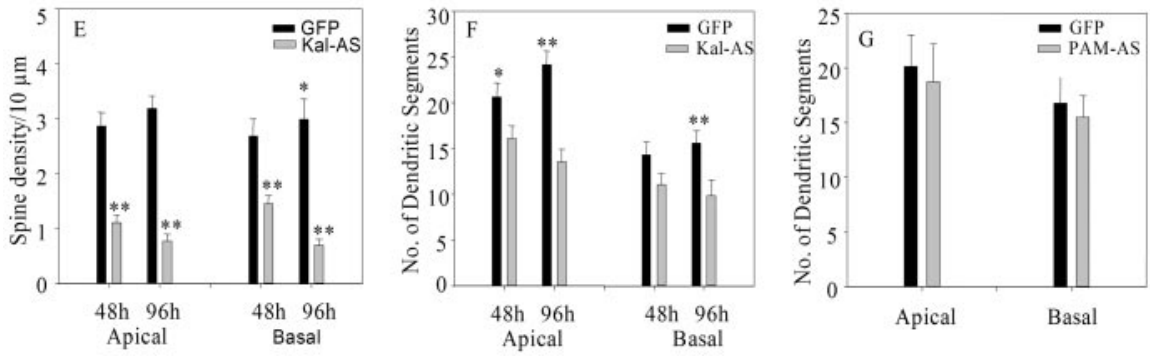

Figure 9. Expression of Kalirin antisense RNA in hippocampal CA1 neurons results in progressive loss of dendritic spines. $A-D$, High-power images (GFP) were taken to evaluate spine density and morphology 48 and $96 \mathrm{hr}$ after transfection of GFP alone ( $A$, C) or GFP plus Kalirin antisense RNA $(B, D)$; representative images of apical dendrites are shown. The long arrow marks a bulbous structure, and short arrows mark thin processes. Scale bar, $10 \mu \mathrm{m}$. E, For both apical and basal dendrites, spine density was quantified after 48 and $96 \mathrm{hr}\left({ }^{* *} p<0.01\right)$. $F$, Dendritic segment analysis showed progressive changes in both apical and basal dendrites $\left({ }^{*} p<0.05 ;{ }^{* *} p<0.01\right)$. G, A similar analysis of neurons expressing PAM antisense RNA showed no significant change from the GFP control after $48 \mathrm{hr}$. (Sala et al., 2001). SPAR, a Rap GAP with two actin-binding domains, a PDZ domain, and a guanylate kinase-like-binding domain, causes spine heads to enlarge, with multilobed shapes (Pak et al., 2001). Overexpression of Drebrin, an actinbinding protein localized to spines, produces longer spines (Hayashi and Shirao, 1999), whereas syndecan-2, a cell surface proteoglycan, accelerates spine maturation (Ethell and Yamaguchi, 1999). Kalirin-7 is one of the few proteins whose primary effect is to increase the number of spines (Penzes et al., 2003). Mice lacking spinophilin, which has an actin-binding site and is a Kal-7 interactor, also have more dendritic spines (Feng et al., 2000).

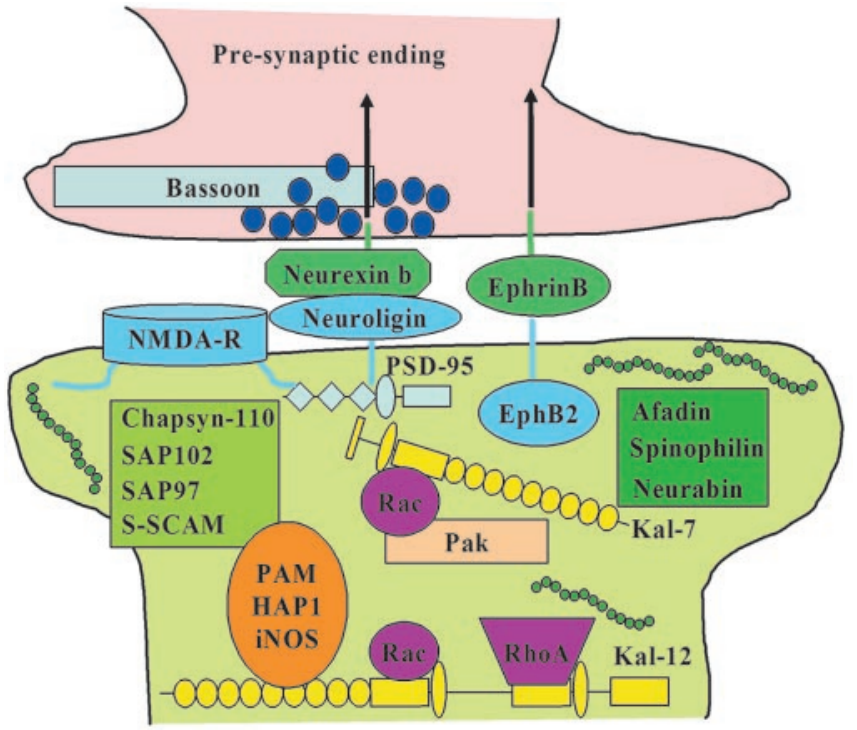

Figure 10. Diagram illustrating the interaction of Kal-7 with three known interactors, PSD 95, Rho proteins, and EphB2. Many different factors clearly regulate both spine formation and dendritic morphology. Kalirin is only one of the many factors that interact to provide precise, minute-to-minute control of these dynamic structures. Nevertheless, elimination of this one gene product results in a profound disruption of the dendritic structure. Other Kal-7 interactors that are MAGUK family members or actin-binding proteins are noted, as are other proteins identified as Kal spectrin interactors. Disruption of these interactions after antisense mediatedreductions in Kalirin expression could lead to the observed loss of spines and decrease in dendritic complexity.

observed may simply reflect loss of innervation of the cell in which Kalirin expression has been reduced (Vaillant et al., 2002).

A number of molecules affect spine shape and size (Pak et al.,
Kalirin is essential for maintenance of dendritic branches

Dendritic branching patterns constitute one determinant of signaling efficiency. Both dendritic growth and branching are regulated by multiple growth factors (Xu et al., 2000; Whitford et al. 2002). Reduced Kalirin expression resulted in decreased dendritic length and complexity, with retraction of apical dendrites preceding retraction of basal dendrites. Although spine structure depends primarily on actin, the stability of the dendritic tree is based on microtubules (Kaech et al., 2001; Whitford et al., 2002). Coordinate control of actin and tubulin is required for normal dendritic growth and maturation, and GEFs that activate RhoG, as Kalirin and Trio do, may have a special role in this process (Vignal et al., 2001). Activation of RhoA and ROCK (Rhokinase), a downstream effector, limits dendritic growth (Lee et al., 2000; Nakayama et al., 2000; Tashiro et al., 2000). The simplification of the dendritic arbor that occurs when Kalirin expression is reduced suggests that Kalirin normally plays a role in limiting RhoA activation. Because Kalirin binds to RhoA but does not activate it (M. R. Schiller, unpublished results), reduced Kalirin expression could release RhoA, making it available for activation and causing dendritic retraction.

\section{How might loss of a single protein cause such dramatic changes?}

Dendritic morphology clearly reflects inputs from multiple sources mediated through many signaling pathways. The fact that eliminating a single player in this complex process has such a profound effect presumably reflects the complex manner in which different inputs are integrated. The various isoforms of Kalirin are large proteins with multiple domains; it is estimated that Kal-12 could span $85 \mathrm{~nm}$, a significant distance in a typical spine head (100-1000 nm diameter) (Nimchinsky et al., 2002). Several proteins localized to dendritic spines are known to inter- 
act with Kalirin, and the combined loss of these multiple interactions may be responsible for the phenotype observed (Figs. 2-9). For the purposes of this discussion, the effects of reducing levels of Kalirin are discussed in terms of disrupting the functions of three known Kalirin interactors, PSD-95, Rho GTPases and EphB2 (Fig. 10).

\section{PSD-95}

PSD-95, a MAGUK localized at glutamatergic synapses, is a scaffold for receptors and cytoskeletal elements (Cho et al., 1992; Garner et al., 2000). Although overexpression of PSD-95 increases spine size, its loss does not affect synaptic structure (ElHusseini et al., 2000). Kal-7 and Kal-12 are partially colocalized with PSD-95 at postsynaptic endings, and Kal-7 complexes directly with PSD-95, resulting in a decrease in its GEF activity for Rac1 (Penzes et al., 2001a). Kal-7 also interacts with other members of the MAGUK family (Chapsyn-110, synapse-associated protein SAP102, SAP97, and synaptic scaffolding molecule S-SCAM), and loss of these interactions could disrupt postsynaptic organization, triggering trans-synaptic changes. PSD-95 communicates with the presynaptic terminal through an intercellular adhesion complex, neuroligin and neurexin (Cantallops and Cline 2000; Rao et al., 2000). A retrograde signal resulting in elimination of Bassoon staining and presynaptic input could be conveyed in this way.

\section{Rho GTPases}

Spines are rich in filamentous actin, the major determinant of spine dynamics and dendritic plasticity (Fischer et al., 1998; Matus, 2000; Hering and Sheng 2001; Kaech et al., 2001). Rho GTPases are key regulators of actin dynamics (Hall, 1998; Luo, 2002), and Rac1, RhoA, and Cdc42 are highly expressed in the hippocampus (Olenik et al., 1997). The first GEF domain of Kalirin activates Rac1, Rac2, RhoG, and RhoC but does not activate RhoA, RhoB, or Cdc42. The second GEF domain of Kalirin does not activate Rac1, Rac2, or RhoA but does activate RhoG and RhoC (Schiller, unpublished results). Constitutively active Rac1 disrupts normal dendritic spine morphology (Nakayama et al., 2000). Activated Rac1 then interacts with p21 activated kinase PAK1 to affect actin organization in spines (Penzes et al., 2003). Genetic studies place dTrio, the Drosophila ortholog of Kalirin, and PAK1 in the same signaling pathway (Newsome et al., 2000). Because spine formation can be blocked by a cell-permeant inhibitor of Pak kinase (Penzes et al., 2003), the ability of Kalirin to activate PAK1 may contribute to its ability to control spine formation (Fig. 10). Spinophilin, neurabin, and afadin each bind filamentous actin and were identified as Kal-7 interactors in a yeast two-hybrid screen; each of these could also contribute to the ability of Kal-7 to control spine formation.

\section{EphB2}

Spine formation is stimulated when $\mathrm{EphB} 2$, a receptor-tyrosine kinase, is activated by its ligand, ephrin B1 (Penzes et al., 2003). Kalirin is an essential component of this signaling pathway, which requires the PDZ-binding domain of Kal-7, the GEF activity of Kalirin, activation of Rac1, and activation of PAK1. A reduction in levels of Kalirin could interfere with this signaling pathway. In addition, because the ephrin B1-EphB2 complex is capable of bidirectional signaling, disruption of this pathway could also be involved in the retrograde signal, resulting in elimination of the presynaptic input. Kal-7 interactions with the PDZ domains of syntenin, afadin, and protein interactor with C-kinase PICK-1 (Penzes et al., 2001a), proteins known to interact with EphB receptors, could also contribute to disruption of this important pathway following reductions in Kalirin expression.

Although its GEF activity is essential for some functions, it is clear that Kalirin exerts a number of GEF-independent actions. Some of these may be of critical importance to the ability of dendrites to generate spines and the maintenance of the dendritic tree. Binding of inactive Rho proteins to a GEF domain of Kalirin may prevent their activation. Kalirin is a large protein, allowing it to interact in a specific manner with proteins such as inducibe nitric oxide synthase, huntingtin-associated protein HAP1, and PAM (Fig. 10). The release of these proteins after antisensemediated decreases in Kalirin could lead to some of the effects observed.

Kalirin is in the right place at the right time to be involved in dendritic branching and spine formation, and our current and previous studies indicate that it does so. Maintenance of dendritic spines and the dendritic arbor reflects the integration of many inputs. Many individual players, such as Kalirin, may prove to play essential roles. Understanding how the many inputs known to play a role use various signaling pathways to produce the final output remains a challenge.

\section{References}

Alam MR, Caldwell BD, Johnson RC, Darlington DN, Mains RE, Eipper BA (1996) Novel proteins that interact with the COOH-terminal cytosolic routing determinants of an integral membrane peptide-processing enzyme. J Biol Chem 271:28636-28640.

Alam MR, Johnson RC, Darlington DN, Hand TA, Mains RE, Eipper BA (1997) Kalirin, a cytosolic protein with spectrin-like and GDP/GTP exchange factor-like domains that interacts with peptidylglycine alphaamidating monooxygenase, an integral membrane peptide-processing enzyme. J Biol Chem 272:12667-12675.

Bloomquist BT, Eipper BA, Mains RE (1991) Prohormone-converting enzymes: regulation and evaluation of function using antisense RNA. Mol Endocrinol 5:2014-2024.

Cantallops I, Cline HT (2000) Synapse formation: if it looks like a duck and quacks like a duck. Curr Biol 10:R620-R623.

Cho KO, Hunt CA, Kennedy MB (1992) The rat brain postsynaptic density fraction contains a homolog of the Drosophila discs-large tumor suppressor protein. Neuron 9:929-942.

Ehlers MD (2002) Molecular morphogens for dendritic spines. Trends Neurosci 25:64-67.

El-Husseini AE, Schnell E, Chetkovich DM, Nicoll RA, Bredt DS (2000) PSD-95 involvement in maturation of excitatory synapses. Science 290:1364-1368.

Estrach S, Schmidt S, Diriong S, Penna A, Blangy A, Fort P, Debant A (2002) The Human Rho-GEF trio and its target GTPase RhoG are involved in the NGF pathway, leading to neurite outgrowth. Curr Biol 12:307-312.

Ethell IM, Yamaguchi Y (1999) Cell surface heparan sulfate proteoglycan syndecan-2 induces the maturation of dendritic spines in rat hippocampal neurons. J Cell Biol 144:575-586.

Feng J, Yan Z, Ferreira A, Tomizawa K, Liauw JA, Zhuo M, Allen PB, Ouimet CC, Greengard P (2000) Spinophilin regulates the formation and function of dendritic spines. Proc Natl Acad Sci USA 97:9287-9292.

Fischer M, Kaech S, Knutti D, Matus A (1998) Rapid actin-based plasticity in dendritic spines. Neuron 20:847-854.

Gainer H, Fields RL, House SB (2002) Neuronal transfection using particlemediated gene transfer, In: Cellular and molecular methods in neurobiological research (Merighi A, Carmignoto G, eds), pp 67-84. Berlin: Springer.

Garner CC, Nash J, Huganir RL (2000) PDZ domains in synapse assembly and signalling. Trends Cell Biol 10:274-280.

Hall A (1998) Rho GTPases and the actin cytoskeleton. Science 279:509-514.

Hansel DE, Quinones ME, Ronnett GV, Eipper BA (2001) Kalirin, a GDP/ GTP exchange factor of the Dbl family, is localized to nerve, muscle, and endocrine tissue during embryonic rat development. J Histochem Cytochem 49:833-844.

Hayashi K, Shirao T (1999) Change in the shape of dendritic spines caused 
by overexpression of drebrin in cultured cortical neurons. J Neurosci 19:3918-3925.

Hering H, Sheng M (2001) Dendritic spines: structure, dynamics and regulation. Nat Rev Neurosci 2:880-888.

Johnson RC, Penzes P, Eipper BA, Mains RE (2000) Isoforms of kalirin, a neuronal $\mathrm{Dbl}$ family member, generated through use of different $5^{\prime}$ - and $3^{\prime}$-ends along with an internal translational initiation site. J Biol Chem 275:19324-19333.

Kaech S, Parmar H, Roelandse M, Bornmann C, Matus A (2001) Cytoskeletal microdifferentiation: a mechanism for organizing morphological plasticity in dendrites. Proc Natl Acad Sci USA 98:7086-7092.

Lee T, Winter C, Marticke SS, Lee A, Luo L (2000) Essential roles of Drosophila RhoA in the regulation of neuroblast proliferation and dendritic but not axonal morphogenesis. Neuron 25:307-316.

Luo L (2000) Trio quartet in D. melanogaster. Neuron 26:1-2.

Luo L (2002) Actin cytoskeleton regulation in neuronal morphogenesis and structural plasticity. Annu Rev Cell Dev Biol 18:601-635.

Ma XM, Lightman SL, Aguilera G (1999) Vasopressin and corticotropinreleasing hormone gene responses to novel stress in rats adapted to repeated restraint. Endocrinology 140:3623-3632.

Ma XM, Johnson RC, Mains RE, Eipper BA (2001) Expression of Kalirin, a neuronal GDP/GTP exchange factor of the Trio family, in the central nervous system of the adult rat. J Comp Neurol 429:388-402.

Ma XM, Mains RE, Eipper BA (2002) Plasticity in hippocampal peptidergic systems induced by repeated electroconvulsive shock. Neuropsychopharmacology 27:55-71.

Mains RE, Bloomquist BT, Eipper BA (1991) Manipulation of neuropeptide biosynthesis through the expression of antisense RNA for peptidylglycine $\alpha$-amidating monooxygenase. Mol Endocrinol 5:187-193.

Maletic-Savatic M, Malinow R, Svoboda K (1999) Rapid dendritic morphogenesis in CAl hippocampal dendrites induced by synaptic activity. Science 283:1923-1927.

Matus A (2000) Actin-based plasticity in dendritic spines. Science 290:754-758.

May V, Schiller MR, Eipper BA, Mains RE (2002) Kalirin Dbl-homology GEF1 domain initiates new axon outgrowths through RhoG-mediated mechanisms. J Neurosci 22:6980-6990.

McPherson CE, Eipper BA, Mains RE (2002) Genomic organization and differential expression of Kalirin isoforms. Gene 284:41-51.

Nakayama AY, Harms MB, Luo L (2000) Small GTPases Rac and Rho in the maintenance of dendritic spines and branches in hippocampal pyramidal neurons. J Neurosci 20:5329-5338.

Newsome TP, Schmidt S, Dietzl G, Keleman K, Asling B, Debant A, Dickson BJ (2000) Trio combines with Dock to regulate Pak activity during photoreceptor axon pathfinding in Drosophila. Cell 101:283-294.

Nimchinsky EA, Sabatini BL, Svoboda K (2002) Structure and function of dendritic spines. Annu Rev Physiol 64:313-353.

O’Brien SP, Seipel K, Medley QG, Bronson R, Segal R, Streuli M (2000) Skeletal muscle deformity and neuronal disorder in Trio exchange factordeficient mouse embryos. Proc Natl Acad Sci USA 97:12074-12078.

Olenik C, Barth H, Just I, Aktories K, Meyer DK (1997) Gene expression of the small GTP-binding proteins RhoA, RhoB, Rac1, and Cdc42 in adult rat brain. Brain Res Mol Brain Res 52:263-269.

Pak DT, Yang S, Rudolph-Correia S, Kim E, Sheng M (2001) Regulation of dendritic spine morphology by SPAR, a PSD-95-associated RapGAP. Neuron 31:289-303.

Paquet L, Massie B, Mains RE (1996) Proneuropeptide Y processing in large dense-core vesicles: manipulation of prohormone convertase expression in sympathetic neurons using adenoviruses. J Neurosci 16:964-973.

Penzes P, Johnson RC, Alam MR, Kambampati V, Mains RE, Eipper BA
(2000) An isoform of kalirin, a brain-specific GDP/GTP exchange factor, is enriched in the postsynaptic density fraction. J Biol Chem 275:6395-6403.

Penzes P, Johnson RC, Sattler R, Zhang X, Huganir RL, Kambampati V, Mains RE, Eipper BA (2001a) The neuronal Rho-GEF Kalirin-7 interacts with PDZ domain-containing proteins and regulates dendritic morphogenesis. Neuron 29:229-242.

Penzes P, Johnson RC, Kambampati V, Mains RE, Eipper BA (2001b) Distinct roles for the two Rho GDT/GTP exchange factor domains of kalirin in regulation of neurite outgrowth and neuronal morphology. J Neurosci 21:8426-8434.

Penzes P, Beeser A, Chernoff J, Schiller MR, Eipper BA, Mains RE, Huganir RL (2003) Rapid induction of dendritic spine morphogenesis by transsynaptic ephrinB-EphB receptor activation of the Rho-GEF kalirin. Neuron 37:263-274.

Quilliam LA, Rebhun JF, Castro AF (2002) (2002) A growing family of guanine nucleotide exchange factors is responsible for activation of Rasfamily GTPases. Prog Nucleic Acid Res Mol Biol 71:391-444.

Rao A, Kim E, Sheng M, Craig AM (1998) Heterogeneity in the molecular composition of excitatory postsynaptic sites during development of hippocampal neurons in culture. J Neurosci 18:1217-1229.

Rao A, Harms KJ, Craig AM (2000) Neuroligation: building synapses around the neurexin-neuroligin link. Nat Neurosci 3:747-749.

Sala C, Piech V, Wilson NR, Passafaro M, Liu G, Sheng M (2001) Regulation of dendritic spine morphology and synaptic function by Shank and Homer. Neuron 31:115-130.

Sala C, Futai K, Yamamoto K, Worley PF, Hayashi Y, Sheng M (2003) Inhibition of dendritic spine morphogenesis and synaptic transmission by activity-inducible protein Homerla. J Neurosci 23:6327-6337.

Sampo B, Kaech S, Kunz S, Banker G (2003) Two distinct mechanisms target membrane proteins to the axonal surface. Neuron 37:611-624.

Schmidt A, Hall MN (1998) Signaling to the actin cytoskeleton. Annu Rev Cell Dev Biol 14:305-338.

Shiraishi Y, Mizutani A, Mikoshiba K, Furuichi T (2003) Coincidence in dendritic clustering and synaptic targeting of Homer proteins and NMDA receptor complex proteins NR2B and PSD95 during development of cultured hippocampal neurons. Mol Cell Neurosci 22:188-201.

Tashiro A, Minden A, Yuste R (2000) Regulation of dendritic spine morphology by the rho family of small GTPases: antagonistic roles of Rac and Rho. Cereb Cortex 10:927-938.

tom Dieck S, Sanmarti-Vila L, Langnaese K, Richter K, Kindler S, Soyke A, Wex H, Smalla KH, Kampf U, Franzer JT, Stumm M, Garner CC, Gundelfinger ED (1998) Bassoon, a novel zinc-finger CAG/glutaminerepeat protein selectively localized at the active zone of presynaptic nerve terminals. J Cell Biol 142:499-509.

Vaillant AR, Zanassi P, Walsh GS, Aumont A, Alonso A, Miller FD (2002) Signaling mechanisms underlying reversible, activity-dependent dendrite formation. Neuron 34:985-998.

Vignal E, Blangy A, Martin M, Gauthier-Rouviere C, Fort P (2001) Kinectin is a key effector of RhoG microtubule-dependent cellular activity. Mol Cell Biol 21:8022-8034.

Wellmann H, Kaltschmidt B, Kaltschmidt C (1999) Optimized protocol for biolistic transfection of brain slices and dissociated cultured neurons with a hand-held gene gun. J Neurosci Methods 92:55-64.

Whitford KL, Dijkhuizen P, Polleux F, Ghosh A (2002) Molecular control of cortical dendrite development. Annu Rev Neurosci 25:127-149.

Xu B, Zang K, Ruff NL, Zhang YA, McConnell SK, Stryker MP, Reichardt LF (2000) Cortical degeneration in the absence of neurotrophin signaling: dendritic retraction and neuronal loss after removal of the receptor TrkB. Neuron 26:233-245. 